\title{
Primary Angioplasty without Stenting for Symptomatic, High-Grade Intracranial Stenosis with Poor Circulation
}

\author{
(D) Y. Wang, (D). Ma, (DP. Gao, (DY. Chen, DB. Yang, and (D) Liao
}

\begin{abstract}
BACKGROUND AND PURPOSE: Although the Stenting versus Aggressive Medical Management for Preventing Recurrent Stroke in Intracranial Stenosis (SAMMPRIS) study demonstrated a high incidence of perioperative complications for Intracranial Atherosclerotic disease (ICAD) treatments with stent placement, some studies have shown that submaximal angioplasty with an undersized balloon limits the risks of perioperative complications, suggesting that intervention may remain an alternative option for ICAD if perioperative complications are minimized. We sought to evaluate clinical and angiographic outcomes after primary angioplasty without stent placement in patients with symptomatic, high-grade intracranial stenosis and poor antegrade flow who were refractory to medical therapy.
\end{abstract}

MATERIALS AND METHODS: All cases with high-grade, symptomatic intracranial stenosis and poor antegrade flow treated with intracranial angioplasty without stent placement at Xuanwu Hospital, Capital Medical University, from January 2010 to December 2016, were retrospectively reviewed. The main outcomes included the changes in antegrade flow and residual stenosis and any stroke or death within 1 month. We also evaluated functional outcomes, stroke, and restenosis in patients on follow-up.

RESULTS: Thirty-five patients (mean age, 64.3 years) were included, and the mean follow-up time was 9.7 months. The average preprocedural stenosis was $88.4 \%$. The immediate, average postprocedure stenosis rate was $25.3 \%$, and the average postprocedural stenosis rate at last angiographic follow-up was $34.7 \%$. The primary end point of major stroke or death at 30 days was observed in 1 patient (1/35, $2.9 \%)$, and no patient had intraprocedural complications. The incidence of stroke or death at the last follow-up was $2.9 \%$, which was superior to the results of the medical and stent-placement arms of the SAMMPRIS study. Severe restenosis was observed in $3(3 / 25,12 \%)$ patients but without any symptoms.

CONCLUSIONS: In this retrospective series, primary balloon angioplasty was an effective treatment option for symptomatic intracranial stenosis with a high risk of stroke.

ABBREVIATION: SAMMPRIS = Stenting versus Aggressive Medical Management for Preventing Recurrent Stroke in Intracranial Stenosis

$T^{3}$

he results of the Stenting versus Aggressive Medical Management for Preventing Recurrent Stroke in Intracranial Stenosis (SAMMPRIS) study showed a 5.8\% 30-day stroke or death rate in the medical treatment arm compared with $14.7 \%$ in the stent arm. ${ }^{1}$ Therefore, the optimal treatment for symptomatic intracranial stenosis remains unclear. Many experts continue to question whether certain population subsets might still benefit from endovascular therapy for treatment of severe intracranial stenosis. There is clearly still room for great improvement in the

Received January 11, 2018; accepted after revision May 7.

From the Department of Neurosurgery, Xuanwu Hospital, Capital Medical University, Beijing, China.

Please address correspondence to Liqun Jiao, MD, PhD, Xuanwu Hospital, Capital Medical University, Beijing 100053, China; e-mail: Liqunjiao@sina.cn

三 Indicates article with supplemental on-line table.

http://dx.doi.org/10.3174/ajnr.A5708 management of these patients because the projected stroke or death rate at 1 year has approached $12.2 \%$, even in the medical arm of the SAMMPRIS study. Thus, for patients at the highest risk of stroke, a more permissive role for primary angioplasty, especially in situations in which stent navigation is technically challenging, has been advocated. ${ }^{2}$

Compared with stent-placement angioplasty, primary angioplasty may have some advantages: First, the stent system is difficult to advance if the target artery is too tortuous and may cause injury to atherosclerotic plaques, such as increasing the risk of the snowplowing effect and the "cheese grater" effect. Several recent reports have suggested there are advantages of primary angioplasty without stent placement for the treatment of intracranial stenosis in selected patients. ${ }^{3}$

In this retrospective review of cases from our single center, we analyzed high-risk patients with stroke treated with primary bal-

AJNR Am J Neuroradiol 39:1487-92 Aug 2018 www.ajnr.org

1487 
loon angioplasty without stent placement to compare the outcomes of these patients with those of the medical and stent placement treatment arms of the SAMMPRIS study as well as with other published series.

\section{MATERIALS AND METHODS \\ Patient Selection}

Patient selection included all patients who underwent primary intracranial balloon angioplasty at Xuanwu Hospital, Capital Medical University, from January 2010 to December 2016. This trial was performed in line with the requirements of the ethics committee, and all patients gave their signed informed consent before the procedure.

All patients underwent DSA to confirm stenosis, and their angiographic characteristics were evaluated. The degree of stenosis was measured by digital subtraction angiography according to the criteria of the Warfarin-Aspirin Symptomatic Intracranial Disease method. ${ }^{4}$ Endovascular therapy was considered after discussion by an expert team on neurovascular diseases, including a neuroradiologist, neurologist, and neurosurgeon. For patients with complicated angiographic features, such as extremely eccentric lesions or lesions close to perforators, angioplasty with stent placement was considered unsuitable or dangerous, and only primary angioplasty without a stent was suggested. The 35 patients who underwent primary angioplasty had high-grade, angiographic stenosis $(>70 \%)$ and poor antegrade flow with ischemic symptoms in the territory of the same vascular distribution, and their angiographic characteristics met at least 1 of the following conditions: a lesion of $>10 \mathrm{~mm}$ in length, extremely eccentric, or near perforators. The Thrombolysis in Cerebral Infarction grading system was used to evaluate the antegrade blood flow according to digital subtraction angiography. The assessment of neuroimaging was performed by an independent neurologist who was blinded to the clinical outcomes of the patients. Patients were excluded if they had acute infarcts within 2 weeks or nonatherosclerotic lesions confirmed by high-resolution MR imaging.

\section{Periprocedural Management}

The procedures were performed by an experienced neurointerventionist at our center. General anesthesia was induced in all patients. After vascular access was achieved, intravenous heparin was administered via a bolus of $75 \mathrm{U} / \mathrm{kg}$ followed by a half dose 1 hour later. The guiding catheter was advanced into the cervical vertebral or internal carotid artery as high as the vessel tortuosity allowed. A 0.014-inch guidewire would be carefully manipulated across the lesion, and the balloon catheter was then used to navigate the microwire. When it was difficult for the microwire to reach the safety zone, usually a microcatheter was also used if the target artery was too tortuous or there was very severe stenosis. The microcatheter would be exchanged for the balloon catheter after it crossed the stenotic lesion. In the exchange procedure, the tip of the microwire was shaped into a $U$ and placed in a straight portion of a distal artery to avoid the possibility of a wire perforation during the procedure. Angioplasty was performed using a balloon approximately not greater than $70 \%$ of the size of the normal vessel diameter. A Gateway balloon (Stryker, Kalamazoo, Michigan) or a Maverick balloon (Boston Scientific, Natick, Massachusetts) was chosen as the device during the procedure as the surgeons' experience dictated. The length of the lesion should have been covered totally by the chosen balloon, and the balloon should have been positioned precisely under the guidance of the roadmap.

The balloon was inflated as slowly as possible during the procedure, usually, $1 \mathrm{~atm}$ per 10 seconds to reach the nominal pressure, and was kept inflated for at least 60 seconds. In addition, the balloon was slowly deflated by $1 \mathrm{~atm}$ per 30 seconds. With a normal pressure of 6 atm as given in the balloon catheter instructions, this procedure accounted for no more than 5-8 minutes of flow cessation per case.

All procedures were performed with the patient under general anesthesia using a standard transfemoral access. Stenosis $<50 \%$ after angioplasty was acceptable. Usually, a single procedure of balloon inflation angioplasty was performed. If residual stenosis was $>50 \%$ or the phenomenon of elastic recoil was distinct, repeat angioplasty would be performed with a higher inflation pressure or with a larger balloon within the range of $80 \%$ of the diameter of the distal normal artery.

A noncontrast head CT was performed to ensure that potential hemorrhage did not occur after the procedure. A glycoprotein IIb/IIIa inhibitor was routinely administered for 12 hours (tirofiban, weight-based dose) after exclusion of hemorrhage by the CT scan. A weight-based dose of low-molecular-weight heparin was given to all patients every 12 hours subcutaneously for 3 days, and patients were monitored closely until discharge. Preoperative and postoperative images of 2 sample cases are presented in the case presentation.

\section{Periprocedural Medical Management}

All patients received aspirin (100 mg/day) and clopidogrel (75 $\mathrm{mg} /$ day) for at least 5 days before the intervention. The dualantiplatelet therapy was maintained for 90 days after the procedure. Risk factor control was suggested to achieve the following: systolic blood pressure of $<140 \mathrm{~mm} \mathrm{Hg}$ (or $<130 \mathrm{~mm} \mathrm{Hg}$ in patients with diabetes mellitus), low-density lipoprotein of $<70$ $\mathrm{mg} / \mathrm{dL}(1.81 \mathrm{mmol} / \mathrm{L})$ or a decrease by $50 \%$, smoking cessation, and lifestyle modifications to reduce obesity and sedentary states.

\section{Clinical and Imaging Assessment}

All patients were systematically assessed independently by an expert team including a senior neurologist and a senior neurosurgeon. Neurologic status was evaluated using the NIHSS, Barthel Index (BI), and mRS on admission and within 1 week before discharging postoperatively. The follow-up evaluation of neurologic status was also performed at 90 days and at 6 and 12 months postoperatively in the outpatient department. The patients' characteristics and risk factors are presented in the Table.

Pre- and postoperative head MR imaging were performed for all patients to evaluate ischemic lesions using T1, T2, and DWI series. MR imaging was assessed independently by an experienced neuroradiologist.

\section{Follow-Up Policy}

All data were collected from clinical records, operation records, and medical imaging systems. We mainly evaluated the degree of stenosis and the changes in antegrade flow pre-/posttreatment 
Patient and lesion characteristics

\begin{tabular}{lc}
\hline \multicolumn{1}{c}{ Characteristic $(\mathbf{N}=\mathbf{3 5})$} & No. (\%) \\
\hline Mean age (yr) & $63.43 \pm 5.41$ \\
Male & $74.3 \%$ \\
Risk factors & \\
$\quad$ Diabetes mellitus & $9(25.7 \%)$ \\
Hypertension & $22(62.8 \%)$ \\
Hyperlipidemia & $14(40 \%)$ \\
Atrial fibrillation & $2(5.7 \%)$ \\
Coronary artery disease & $7(20 \%)$ \\
Lesion location & \\
Basilar artery & 16 \\
Supraclinoid ICA & 2 \\
M1 segment of MCA & 5 \\
M2 segment of MCA & 4 \\
Vertebral artery & 2 \\
Vertebrobasilar junction & 6 \\
\hline
\end{tabular}

and the adverse events related to the interventional therapy to clarify its safety. To appraise its long-term efficacy, we also assessed the patients' functional status, recurrence of stroke, rate of restenosis, and reinterventions for the lesion. A modified Rankin Scale score of 0-2 was defined as good functional status. Follow-up imaging by digital subtraction angiography and/or MRA or CTA was performed to assess the rate of restenosis (restenosis was defined as $>50 \%$ ), and head MR imaging was used to look for recurrent ischemic lesions 6 months after the intervention. Retreatment was considered when the patient had recurrent ischemic symptoms and $>50 \%$ restenosis occurred.

\section{Illustrative Cases}

Example 1. A 65-year-old man had intermittent vertigo for 3 months, left-sided weakness, and slurred speech. Even with aggressive medical therapy including aspirin (100 mg/day) and clopidogrel (75 mg/day) plus a statin for 3 months, he still had vertigo. MR imaging revealed a pons infarction, and DSA demonstrated severe stenosis of the proximal basilar artery. High Resolution Magnetic Resonance Imaging revealed the site of the stenosis. Regarding the angiographic characteristics of the lesion, the length of the lesion was approximately $11 \mathrm{~mm}$ and included the site of the vertebrobasilar junction, and the right AICA was very close to the stenotic lesion. He underwent primary angioplasty for the vertebrobasilar junction stenosis, and the postoperative period was uneventful. The immediate DSA after angioplasty demonstrated that antegrade blood flow was markedly improved, and 2-day postoperative MR imaging showed no fresh infarction in the territory of the posterior circulation. The 14-month follow-up by DSA and MR imaging demonstrated no obvious restenosis and no recurrent infarction. All images are shown in Fig 1.

Example 2. A 63-year-old man had paroxysmal dizziness, numbness of the right hand, and weakness of the lower limb. MR imaging revealed a subacute cerebral infarction involving the left frontal area. DSA identified severe stenosis of the left middle cerebral artery (1 branch of M2), and HRMRI revealed high-grade focal stenosis within the M2 segment of the left MCA. He underwent primary angioplasty of the left MCA. Postoperative DSA showed the lumen obviously improved, and MR imaging indicated no fresh infarction. The 9-month follow-up DSA indicated no obvious restenosis. He was referred to our institution where he under-
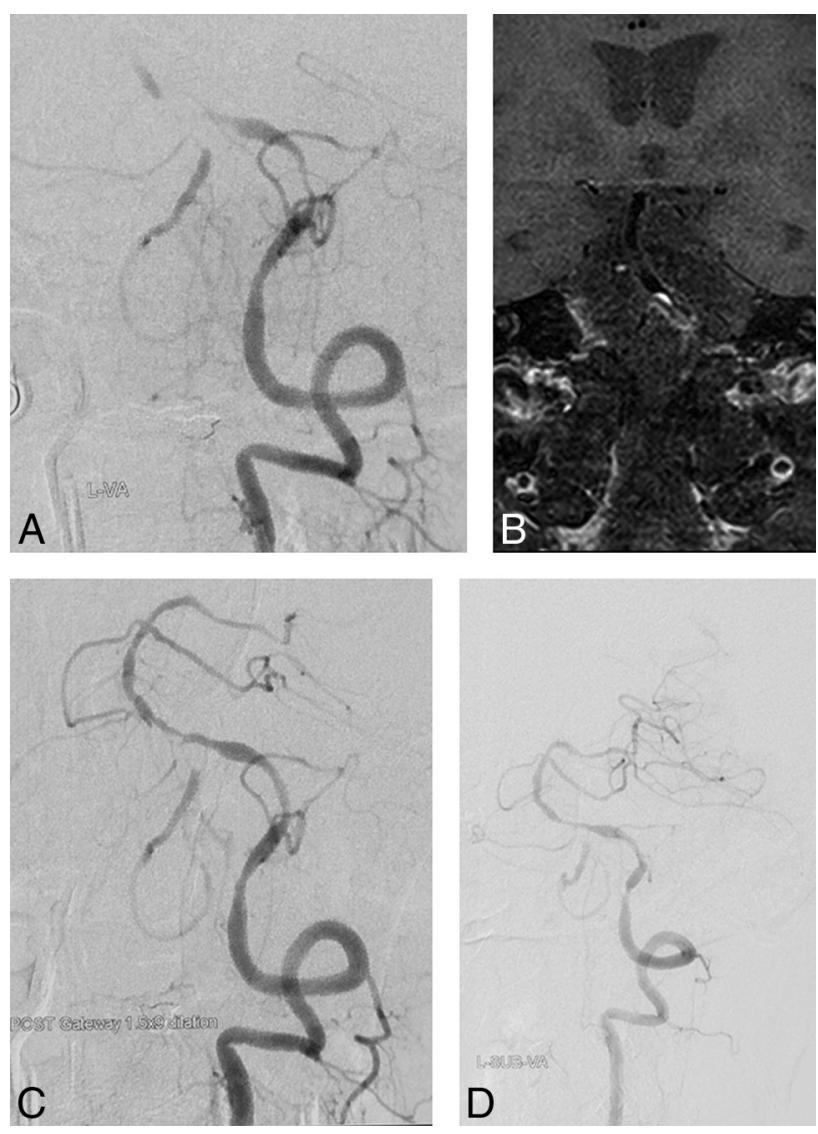

FIG 1. A 65-year-old man with symptomatic severe basilar artery stenosis, refractory to medical therapy (case 1). A, Angiogram of the left vertebral artery reveals severe stenosis of the vertebrobasilar junction (angiographic characteristic: TICI 1). B, High-resolution MR imaging confirms the atherosclerotic lesion and shows the eccentric plaque located at the vertebrobasilar junction and the surface of the plaque enhancement. $C$, Immediate angiogram after primary angioplasty with a 1.5- to 9-mm Gateway balloon; antegrade flow improved and reached $\mathrm{TICl}$ grade $2 \mathrm{~b}$. D, Angiogram of the left vertebral artery at 14-month follow-up reveals no severe restenosis, and antegrade flow reaches $\mathrm{TICl}$ grade 3 .

went angiography and subsequent angioplasty. All images are shown in Fig 2.

\section{RESULTS}

\section{Patients and Intervention Procedures}

Thirty-five patients ( 26 men, 9 women; mean age, $63.43 \pm 5.41$ years; range, 56-74 years) with symptomatic intracranial stenosis were treated by primary balloon angioplasty from January 2010 to December 2016, with a mean of 10.6 months of follow-up. Patients who were treated in the acute period ( 2 weeks from onset) were not included in this study. Most $(n=33)$ of the included patients $(94.3 \%)$ presented with ischemic strokes, and the other 2 patients presented with TIAs (5.7\%). The time interval from the onset of the last symptom to interventional treatment was 35.6 days (range, 15-69 days). The most common risk factor for intracranial stenosis in the study population was hypertension (62.8\%). Other risk factors were as follows: hyperlipidemia (40\%), diabetes mellitus $(25.7 \%)$, coronary artery disease $(20 \%)$, and atrial fibrillation $(5.7 \%)$. The clinical features of all patients in our study are listed in the Table. The anatomic location of the stenotic lesion in each case is shown in the On-line Table. 

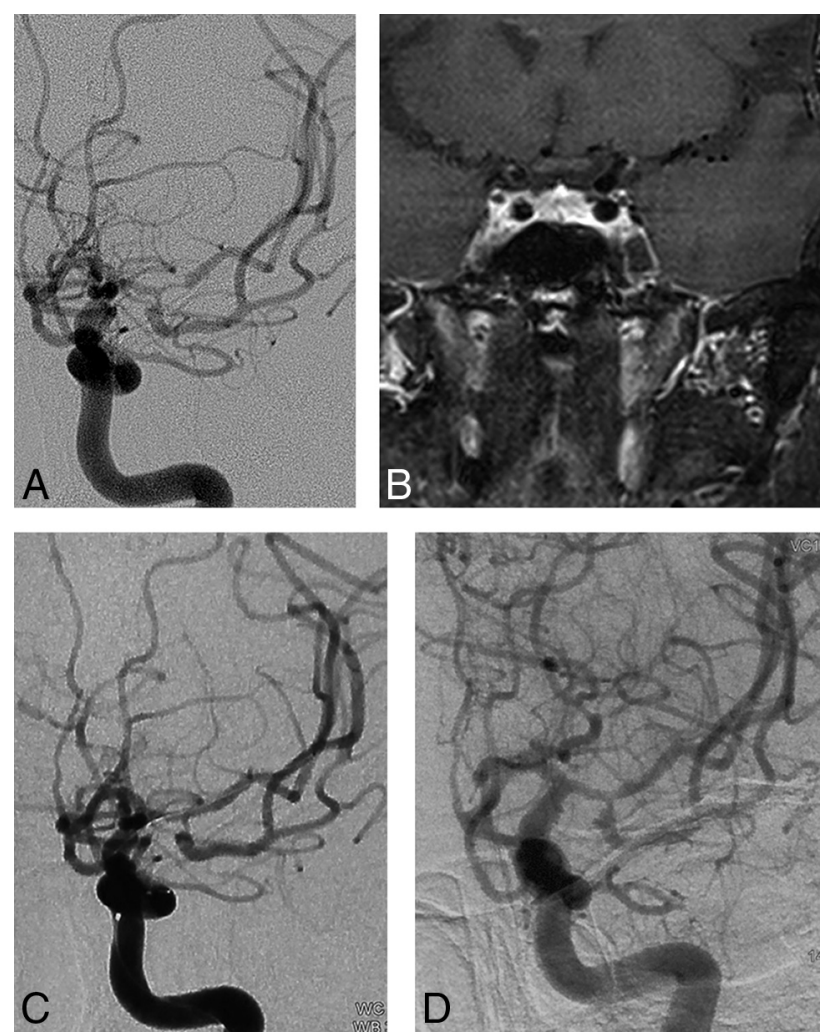

FIG 2. A 63-year-old man with symptomatic MCA severe stenosis. (case 26). A, Angiogram of the left internal carotid artery reveals severe stenosis of the left middle cerebral artery (M2 segment) (angiographic characteristic: $\mathrm{TICl} 2 \mathrm{2a}$ ). $B$, High-resolution MR imaging confirms the atherosclerotic lesion and shows the plaque located at the M2 branch. $C$, Immediate angiogram after primary angioplasty with a 2- to 9-mm Gateway balloon reveals that antegrade flow improved and reached $\mathrm{TICl}$ grade $2 \mathrm{~b}$. $D$, Angiogram of the left vertebral artery at 6-month follow-up demonstrates no severe restenosis, and antegrade flow reached $\mathrm{TICl}$ grade 3 .

In our series, coronary balloon catheters (Maverick) for intracranial angioplasty were used in 2 patients, and Gateway balloon catheters were used in 33 patients. Each balloon catheter was strictly selected to be no larger than $70 \%$ of the normal diameter of the parent vessel. The balloon catheters were dilated slowly to reach the nominal pressure following the manufacturer's instructions. No vessel rupture or wire perforations occurred during the procedures. No stent angioplasty was needed to maintain the patency of the lesions during the procedure.

The average preprocedural stenosis was $82.7 \%$ (range, $71 \%$ $94 \%$ ), and the average immediate postprocedural stenosis was $31.2 \%$ (range, $23 \%-46 \%$ ).

Retreatment was required in only $1(1 / 35,2.9 \%)$ patient during the perioperative period, secondary to acute basilar artery thrombosis 5 days after the procedure. Acute recanalization of the basilar artery was then performed, and the patient recovered well. The primary end point of major stroke or death at 30 days was observed in only this patient $(2.9 \%)$, and no intraprocedural complication occurred.

The mean follow-up was 17.6 months (range, 6-42 months) for 33 patients. Of these 33 patients, 31 (93.9\%) had good nerve function status (mRS, $0-2$ ). Two patients had recurrent ischemic stroke, including one with ipsilateral lacunar infarction (MCA stenosis, 6 months after the interventional therapy) and one with a brain stem infarction (basilar artery stenosis, 14 months after the interventional therapy). The rate of recurrence of ischemic stroke beyond 30 days was $3.0 \%$ at 1 year (1/33 of the followed patients), and the combined rates of major stroke and death within 30 days and the ipsilateral ischemic stroke rate beyond 30 days at 1 year were $6 \%$.

Follow-up imaging data were available in 25 patients, of whom $3(12 \%)$ had restenosis without any symptoms. Of the 33 patients followed, 25 patients underwent some type of radiographic follow-up performed using either conventional angiography, CTA, or MRA. Twenty patients underwent DSA as a primary method of follow-up, 3 patients underwent CTA, and 2 patients underwent MRA as their primary method of follow-up, with an average follow-up interval of 16.3 months (range, 6-38 months). Severe restenosis was observed in $3(3 / 25,12 \%)$ patients, but no symptoms were observed. Retreatment was not performed for these 3 patients because no further symptoms occurred.

\section{DISCUSSION}

Good collateral compensation is seen as a protective factor to reduce the risk of stroke in patients with symptomatic intracranial stenosis, and adequate antegrade flow is associated with favorable outcomes and a lower recurrence of stroke. ${ }^{5}$ Thus, patients with severe intracranial stenosis and poor antegrade blood flow are at high risk of ischemic stroke, and any effort to increase antegrade flow and improve lumen augmentation should reduce the risk of ischemic stroke. Our findings may cause renewed interest in primary balloon angioplasty in this specific patient population with severe stenosis and poor antegrade flow. We report the outcomes of 35 consecutive patients with symptomatic intracranial artery stenosis with poor antegrade circulation who underwent primary angioplasty in this study.

In our case series the 30-day stroke or death rate was $2.9 \%$. The combined stroke or death rate within 1 year beyond 30 days was $6 \%$, and $93.9 \%$ of the available follow-up patients had a good functional status (mRS, $0-2$ ). Because our case series was retrospective, it is difficult to perform direct comparisons with the SAMMPRIS study. However, the results within 30 days in our study were better than those reported in the stent-placement group of the SAMMPRIS study (2.9\% versus $14.7 \%$ ), and the 1 -year results in our study were still better than the results in the stent-placement group of the SAMMPRIS study (6\% versus $19.7 \%)^{6}$

In the SAMMPRIS study, most of the adverse events occurred in the perioperative period, and some perioperative complications were related to the procedure of deploying or manipulating the stent system, such as perforator ischemia, stent thrombosis, and wire perforation. A reappraisal of primary angioplasty without stents has recently been conducted. ${ }^{7}$ There are several potential benefits to primary balloon angioplasty over stent placement in the intracranial circulation. Tortuous anatomy may cause technical challenges in stent navigation and increase procedural risks. Theoretically, the technique of submaximal angioplasty without stents could result in adequate lumen augmentation for a symptomatic stenotic lesion. This technique could significantly avoid 
the danger of arterial injury by the stent system, perforator snow plowing occlusion, and distal embolization. It also may reduce the risk of severe intracerebral hemorrhage caused by hyperperfusion syndrome due to the gradual recovery of the vessel lumen. ${ }^{8}$

Different intracranial stenosis locations have various perioperative complications due to their anatomic features, especially the MCA and basilar artery because these 2 locations involve a perforator-rich zone. In our series, most of these lesions were in the basilar artery, and the subgroup with lesions in the basilar artery had the lowest risk of stroke during medical therapy in SAMMPRIS. However, even the 2-year primary end point of the basilar artery subgroup in SAMMPRIS was 10\% for the medical therapy arm, ${ }^{9}$ which means that these patients still suffered from recurrent stroke, which suggests that intervention may remain an alternative option if perioperative complications are minimized. In our experience, patients with higher grade stenosis and poor antegrade flow are most likely to benefit from flow augmentation by primary angioplasty.

As some literature has mentioned, one of the design flaws of SAMMPRIS was that the time interval between qualifying symptoms and intervention was too short. ${ }^{10}$ In our case series, the time interval from onset of the last symptom to interventional treatment was 35.6 days. The good results of the Wingspan Stent System Post Market Surveillance Study (WEAVE ${ }^{\mathrm{TM}}$ Trial) also prolonged the time interval, which hints that the longer interval may be a protective factor for the intervention.

Our series demonstrates the feasibility and relative safety of primary balloon angioplasty and supports the incorporation of this technique into future trials that seek to identify high-risk population subsets that might benefit from angioplasty in conjunction with aggressive medical therapy for high-grade, symptomatic intracranial atherosclerosis. Our results suggest that primary balloon angioplasty is safer than stent angioplasty in some specific situations.

The contemporary literature includes several reports of primary balloon angioplasty for intracranial atherosclerosis that use a wide variety of balloons, mostly marketed for coronary use, that have reported a success rate and risk profile that are largely comparable with those of stent placement. A recent multi-institutional retrospective review reported a $92 \%$ success rate for primary balloon angioplasty and a 3-month stroke or death rate of $8.5 \%$ in a series of 74 patients. Either Gateway or Maverick balloon catheters were used in all cases, and the mean pretreatment stenosis rate was $79 \% \pm 14 \% .{ }^{11}$ One of the largest multicenter studies to compare primary angioplasty with stent placement for symptomatic intracranial stenosis showed no difference in survival at a 2-year follow-up. The 30-day rate of major stroke or death was $8.4 \%$ in the angioplasty group (8/95) versus $9.2 \%$ in the stent-placement group (9/98). ${ }^{12}$

In our series of 35 patients treated during the past 6 years at a single institution, we found a $2.9 \%$ rate of major stroke or death at 1 month, which is comparable with rates in the existing literature on this topic. The 1-year stroke or death rate in our series was $6 \%$, which was comparable with the 1-year stroke or death rate in the medical arm of the SAMMPRIS study. This raises the question of whether angioplasty confers an up-front risk with a protective payoff from stroke as follow-up time increases. Therefore, further studies regarding the long-term durability of this treatment method are needed.

In our series, a submaximal angioplasty technique with slow inflation was used. No rescue stent placement was necessary because there was no plaque disruption or obvious dissection.

As we continue to add to the collective experience with primary balloon angioplasty for intracranial stenosis, it will be important to study whether certain patient populations or lesions with particular angiographic characteristics are more favorable to interventional treatment.

The senior author routinely administers a glycoprotein IIb/ IIIa inhibitor (eg, eptifibatide [Integrillin]) intraprocedurally. It is possible that the use of this agent may decrease the rate of acute thrombus formation. This effect may be especially pertinent in patients who exhibit some degree of resistance to aspirin, clopidogrel, or both because some studies have shown that this population is at a higher risk of thromboembolic complications from neurointerventions. ${ }^{13}$ This practice is not standardized, and no studies have documented the impact of glycoprotein IIb/IIIa inhibitor use at the time of angioplasty on patient outcomes. Thus, there is a concern regarding increased hemorrhage rates in patients receiving these agents; however, none of the patients in our series experienced a hemorrhage as a result of the procedure.

Primary angioplasty has 2 main disadvantages: iatrogenic arterial dissection and elastic recoil. If arterial occlusion occurs due to dissection or significant residual stenosis, stent placement angioplasty is an effective remedial measure.

For the endovascular therapy of intracranial stenosis, the most important factor is to reduce any perioperative complications. According to our experience, submaximal primary angioplasty may offer a safe treatment alternative to best medical therapy in complex intracranial arterial stenosis. The strict selection of patients is also important. Patients with severe stenosis combined with poor antegrade flow are at a high risk of recurrent stroke if treated with only medical therapy; therefore, it is worth trying an additional interventional therapy for those patients refractory to medical therapy with poor antegrade flow. While it has been suggested that lesion length, degree of stenosis, and anatomic location might also affect endovascular outcomes, the role that these factors play remains largely unknown. If we use the classification of Mori et $\mathrm{al}^{14}$ for intracranial stenosis lesions, type A lesions ( $\leq 5$ $\mathrm{mm}$ in length, concentric plaque) correspond to better clinical outcomes compared with the other 2 types of lesions.

Measuring vessel stenosis is a gross simplification for endovascular therapy. In our study, we took into account antegrade flow by means of the TICI classification. Assessment of angiographic features may be valuable for screening out the subset of patients who are at a higher risk for recurrent stroke due to inadequate flow from an atherosclerotic stenotic lesion. Further prospective studies considering this evaluation for intracranial artery stenosis should be performed to identify which patients are best suited for revascularization.

Due to the inevitable restrictions in this retrospective study, there are still many deficiencies, such as a small sample and the absence of a valid comparison arm. A well-designed randomized controlled study is necessary to verify the safety and efficacy of this treatment for the selected patient group.

AJNR Am J Neuroradiol 39:1487-92 Aug 2018 www.ajnr.org 


\section{CONCLUSIONS}

In our retrospective series, primary angioplasty was found to have a certain value with a relatively acceptable result for symptomatic patients with intracranial stenosis with a high risk of stroke. Further studies may elucidate specific clinical factors, anatomic sites, or patterns of stenosis that make a patient particularly amenable to balloon angioplasty.

Based on our preliminary results, compared with the results of the stent placement arm in the SAMMPRIS study, primary angioplasty for ICAD in the selected patients was a feasible and safe option with a favorable result. The positive effects of primary angioplasty were maintained with a mean of 10.6 months follow-up among the successfully followed patients in our series. In our experience, for patients with symptomatic intracranial stenosis refractory to aggressive medical therapy and for those with poor antegrade circulation, primary angioplasty without a stent should be the first neurointerventional therapy tried before considering stent angioplasty. A prospective randomized controlled trial will be conducted to verify the safety and efficacy of this interventional technique.

\section{REFERENCES}

1. Chimowitz MI, Lynn MJ, Derdeyn CP, et al; SAMMPRIS Trial Investigators. Stenting versus aggressive medical therapy for intracranial arterial stenosis. N Engl J Med 2011;365:993-1003 CrossRef Medline

2. Qureshi AI, Al-Senani FM, Husain S, et al. Intracranial angioplasty and stent placement after stenting and aggressive medical management for preventing recurrent stroke in intracranial stenosis (SAMMPRIS) trial: present state and future considerations. J Neuroimaging 2012;22:1-13 CrossRef Medline

3. Karanam LSP, Sharma M, Alurkar A, et al. Balloon angioplasty for intracranial atherosclerotic disease: a multicenter study. J Vasc Interv Neurol 2017;9:29-34

4. Samuels OB, Joseph GJ, Lynn MJ, et al. A standardized method for measuring intracranial arterial stenosis. AJNR Am J Neuroradiol 2000;21:643-46

5. Lau AY, Wong EH, Wong A, et al. Significance of good collateral compensation in symptomatic intracranial atherosclerosis. Cerebrovasc Dis 2012;33:517-24 CrossRef Medline

6. Derdeyn CP, Chimowitz MI, Lynn MJ, et al. Aggressive medical treatment with or without stenting in high-risk patients with intracranial artery stenosis (SAMMPRIS): the final results of a randomised trial. Lancet 2014;383:333-41

7. Siddiq F, Vazquez G, Memon MZ, et al. Comparison of primary angioplasty with stent placement for treating symptomatic intracranial atherosclerotic diseases: a multicenter study. Stroke 2008;39: 2505-10 CrossRef Medline

8. Dumont TM, Sonig A, Mokin M, et al. Submaximal angioplasty for symptomatic intracranial atherosclerosis: a prospective Phase 1 study. J Neurosurg 2016;125:964-71 CrossRef Medline

9. Derdeyn CP, Chimowitz MI, Lynn MJ, et al; Stenting and Aggressive Medical Management for Preventing Recurrent Stroke in Intracranial Stenosis Trial Investigators. Aggressive medical treatment with or without stenting in high-risk patients with intracranial artery stenosis (SAMMPRIS): the final results of a randomised trial. Lancet 2014;383:333-41 CrossRef Medline

10. Abou-Chebl A, Steinmetz H. Critique of "Stenting versus aggressive medical therapy for intracranial arterial stenosis" by Chimowitz, et al New England Journal of Medicine. Stroke 2012;43:616-20 CrossRef Medline

11. Nguyen TN, Zaidat OO, Gupta R, et al. Balloon angioplasty for intracranial atherosclerotic disease: periprocedural risks and shortterm outcomes in a multicenter study. Stroke 2011;42:107-11 CrossRef Medline

12. Khan M, Naqvi I, Bansari A, et al. Intracranial atherosclerotic disease. Stroke Res Treat 2011;2011:282845 CrossRef Medline

13. Ryu DS, Hong CK, Sim YS, et al. Anti-platelet drug resistance in the prediction of thromboembolic complications after neurointervention. J Korean Neurosurg Soc 2010;48:319-24 CrossRef Medline

14. Mori T, Fukuoka M, Kazita K, et al. Follow-up study after intracranial percutaneous transluminal cerebral balloon angioplasty. AJNR Am J Neuroradiol 1998;19:1525-33 Medline 